\title{
Characteristics of Local Modulation Beam Propagating through Spatial Filter System
}

\author{
Kewei You $\mathbb{D}^{1,2,3}$ Yanli Zhang, ${ }^{1,2}$ Xuejie Zhang, ${ }^{1,2}$ and Jianqiang Zhu $\mathbb{D}^{1,2}$ \\ ${ }^{1}$ Shanghai Institute of Optics and Fine Mechanics, Key Laboratory of High Power Laser and Physics, Chinese Academy of Sciences, \\ Shanghai 201800, China \\ ${ }^{2}$ National Laboratory on High Power Laser and Physics, Shanghai 201800, China \\ ${ }^{3}$ University of Chinese Academy of Sciences, Beijing 100049, China
}

Correspondence should be addressed to Jianqiang Zhu; jqzhu@mail.shcnc.ac.cn

Received 5 February 2017; Revised 22 March 2017; Accepted 13 April 2017; Published 1 January 2018

Academic Editor: Jörg Fink

Copyright (C) 2018 Kewei You et al. This is an open access article distributed under the Creative Commons Attribution License, which permits unrestricted use, distribution, and reproduction in any medium, provided the original work is properly cited.

As local defects may significantly harm beam quality and affect safe operation, a systematic analysis of the ability of a spatial filter to alleviate these adverse effects is required. Thus, the evolutional characteristics of a beam modulated by a local defect propagating through a spatial filter system at an image reply plane and a downstream plane are analyzed in detail. Modulation stripes appear at the image reply plane; these are caused by the pinhole cutoff effect. The modulation degree increases with increasing defect size. The maximum intensification factor can reach 3.2 under certain conditions. Thus, the defect size should be restricted to a reasonable size for safe operation with a specified pinhole size. Moreover, a maximal value appears at the downstream plane, and the intensity enhances with increasing defect size. To ensure beam quality, the maximum allowable defect size and angle of the spatial filter should meet special constraints. The maximum allowable defect size is calculated based on practical configuration parameters.

\section{Introduction}

In a high-power laser system, the laser beam passes through numerous optical components. The amplitude and phase modulations caused by damage defects and contamination particles can result in downstream intensification, which can damage certain optical components in the systems [1-5]. As one of the crucial factors restricting the output power of high-power laser systems, the research of defects in optical components has aroused great interest for a long time. To reduce the detrimental effects of optical component defects, several effective measures have been put into force, such as developing new optical component processing technology and strengthening environmental cleanliness $[6,7]$. On the other hand, the spatial filter serves as an essential part of a high-power laser system [8-11]. An appropriate filter pinhole has been selected to filter out high-frequency modulation, sequentially sharply minimizing nonlinear selffocusing effect $[12,13]$. Although the function of a spatial filter to control beam quality has been investigated extensively, previous studies have concentrated mainly filtering effect with whole beam phase or amplitude distortion, such as large-scale phase aberrations related to intensity distribution [10], wave front defects involving spatial periods [14], and flat-topped multi-Gaussian beams with apertures [15]. There are still some researches about controlling features of spatial filter on nonlinear growth with small soft Gaussian scatterers perturbation [13], but little work is done about local circular defects which is commonly found from the near-field measurement in practical systems.

It is well known that local diffraction modulation may cause a great threat to expensive downstream optical components, so much attention should be paid to how the modulated beam is suppressed by transmitting a spatial filter. Since the local modulation beam may contain a lowfrequency component and has a wider spectrum than an unmodulated beam, the characteristics of local modulation beam propagation through a spatial filter deserve to be discussed in detail. In this paper, a systematic analysis of the propagating characteristics for an image and subsequent 


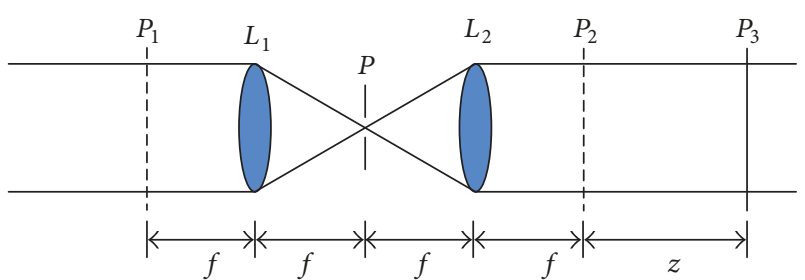

FIGURE 1: Spatial imaging configuration.

plane is conducted. Based on this analysis, some specific cleanliness rules about amplifier and optical components are put forward, thereby providing useful guidance for the inspection of optical components.

\section{Theoretical Model}

Figure 1 depicts a typical spatial imaging configuration that uses the imaging properties of confocal lens pairs. According to a practical spatial filter system in a multipass main amplifier system, the focus lengths of lens $L_{1}$ and $L_{2}$ are both $f$. The distance from input plane $P_{1}$ to $L_{1}$ and the distance from image reply plane $P_{2}$ to $L_{2}$ are also $f$. $P$ represents the pinhole plane and the Fourier spectrum plane of the input field. $P_{3}$ is the observation plane, and the distance from $P_{2}$ is $z$.

According to the basic theory of Fourier Optics, the input and output light fields of an ideal spatial filter have the following relation:

$$
\begin{aligned}
E_{2}\left(x_{2}, y_{2}\right) & =\mathfrak{J}^{-1}\left[T\left(\sqrt{x_{p}^{2}+y_{p}^{2}}\right) \mathfrak{I}\left(E_{1}\left(x_{1}, y_{1}\right)\right)\right] \\
T\left(\sqrt{x_{p}^{2}+y_{p}^{2}}\right) & = \begin{cases}1 & \sqrt{x_{p}^{2}+y_{p}^{2}} \leq \frac{d}{2} \\
0 & \sqrt{x_{p}^{2}+y_{p}^{2}}>\frac{d}{2},\end{cases}
\end{aligned}
$$

where $E_{1}$ and $E_{2}$ are light fields at the input plane and image relay plane; $T$ is the transmission function of the filter pinhole; $d$ is the diameter of the filter pinhole; $x_{1}, y_{1}, x_{2}, y_{2}$, $x_{p}$, and $y_{p}$ are the coordinates at the input plane, image relay plane, and filter pinhole plane, respectively, and operators $\mathfrak{I}$ and $\mathfrak{\Im}^{-1}$ represent the Fourier transform and inverse Fourier transform, respectively. Suppose the input beam is modulated by a local circular defect, and the defect is located at the center of the input beam. Then, the distribution can be expressed as

$$
\begin{aligned}
& E_{1} \\
& \qquad U_{1}\left(x_{1}, y_{1}\right) \\
& \quad+\left(-U_{1}\left(x_{1}, y_{1}\right)+U_{1}\left(x_{1}, y_{1}\right) \times \exp (-i \phi)\right) \\
& \quad \times \operatorname{circ}\left(\sqrt{x_{1}^{2}+y_{1}^{2}}\right) \\
& \operatorname{circ}\left(\sqrt{x_{1}^{2}+y_{1}^{2}}\right)= \begin{cases}1 & \sqrt{x_{1}^{2}+y_{1}^{2}} \leq R \\
0 & \sqrt{x_{1}^{2}+y_{1}^{2}}>R .\end{cases}
\end{aligned}
$$

As shown in (3), the input beam can be represented as the superposition of the background beam and a local supplement beam. The background beam can be treated as a largeaperture uniform light field $\left(U_{1}\left(x_{1}, y_{1}\right)\right)$. The supplement beam is caused by a defect with radius $R$, and $\phi$ denotes the phase modulation. On the basis of the Babinet theorem, the analysis of a beam modulated by a small defect is related to the circular aperture diffraction. Thus, the analysis of the circular aperture diffraction is made first. The distribution at the filter pinhole plane is given by

$$
\begin{aligned}
E^{\prime}\left(x_{p}, y_{p}\right) & =\mathfrak{\Im}\left(E_{1}^{\prime}\left(x_{1}, y_{1}\right)\right) \\
& =\frac{\pi R^{2} A_{1}^{\prime}}{j \lambda f}\left[2 \frac{J_{1}\left(k r_{p} R / f\right)}{k r_{p} R / f}\right],
\end{aligned}
$$

where $E_{1}^{\prime}\left(x_{1}, y_{1}\right)=A_{1}^{\prime}\left(\sqrt{x_{1}^{2}+y_{1}^{2}} \leq R\right)$, the radius of circular aperture is also $R, r_{p}$ is the radial coordinate at the filter pinhole plane, and only intensity is considered. According to (1), the circular aperture diffraction light field at the image relay plane can be written as

$$
\begin{aligned}
E_{2}^{\prime}\left(x_{2}, y_{2}\right)= & \frac{1}{j \lambda f} \iint E^{\prime}\left(x_{p}, y_{p}\right) \\
& \times \exp \left(-\frac{j k x_{p} x_{2}}{f}-\frac{j k y_{p} y_{2}}{f}\right) d x_{p} d y_{p} .
\end{aligned}
$$

According to (3), the light field at the image relay plane can be given by

$$
\begin{aligned}
E_{2}( & \left.x_{2}, y_{2}\right) \\
= & U_{2}\left(x_{2}, y_{2}\right) \\
& \quad+\left(-E_{2}^{\prime}\left(x_{2}, y_{2}\right)+E_{2}^{\prime}\left(x_{2}, y_{2}\right) \times \exp (-i \phi)\right),
\end{aligned}
$$

where $U_{2}\left(x_{2}, y_{2}\right)$ is the imaging light field of the background beam and the other represents the imaging light field of the local supplement beam.

\section{Numerical Simulations and Result Analysis}

Spatial filtering can effectively remove the fastest-growing high-frequency components of the instabilities by using the cutoff frequency provided by the filter pinhole. The diameter of a pinhole in a spatial filter system can be expressed as [17]

$$
d=2 f \theta
$$

where $\theta$ is the angular radius of the focal spot relative to the center of the lens. The cutoff frequency of a spatial filter is $K_{c}=\pi d / \lambda f$ [13]. Thus, the frequency that is higher than $K_{c}$ will be filtered out, making the propagating beam smooth. Combining with (8), $K_{c}=2 \pi \theta / \lambda$. Thus, the frequency that can propagate through the filter pinhole really depends on $\theta$.

Numerical calculations are carried out given that the simulated tenth-order super-Gaussian beam at $1053 \mathrm{~nm}$ has a radius of $185 \mathrm{~mm}$ at $1 \%$ of the maximum intensity. The 
focal length of the lens is $11350 \mathrm{~mm}$, and defects with pure phase modulation $\pi$ are in the range of $R=0.5-3.5 \mathrm{~mm}$. $\theta$ is $200 \mu \mathrm{rad}$, and the diameter of the filter pinhole is $4.6 \mathrm{~mm}$. The intensification factor in a beam section at $z$ is defined by the intensity ratio $I / I_{0} . I$ is the maximum intensity at the observation plane, and $I_{0}$ represents the input intensity on the axis.

Based on the expression of the Airy disk width at the focal plane of the lens $D=1.22 \lambda f / w, w$ is the radius of the transmission beam. We can see that the width is inversely proportional to the radius. Then, the large-aperture beam propagates through the filter pinhole with little distortion and possesses a narrow spectrum. However, spectrum broadening resulting from a small local defect will be cut off by the filter pinhole, causing intensity fluctuations. We performed numerical simulations on beam characteristics at the image relay plane according to (7). Affected by local defects, a nonuniform intensity distribution appears at the image relay plane, which is mainly concentrated in the region of the imaging light field of the local supplement beam. Then, we define this region as a modulation region. Figure 2 presents the intensification factor varying with increasing defect radius. It is found that when the defect radius is $3.2 \mathrm{~mm}$, the Airy disk width of the local supplement beam is approximately $4.6 \mathrm{~mm}$, and the intensification factor grows to a maximum of 3.2. It is clearly seen that the sizes of the Airy disk and pinhole $(\theta=200 \mu \mathrm{rad})$ are basically equal. Therefore, when the cutoff of the pinhole is just at the first notch of the Airy diffraction pattern of the local supplement beam, the imaging light fields of the background beam and the local supplement beam may produce a strong interaction with each other, resulting in the enhanced growth of intensity fluctuations. However, when $R<3.2 \mathrm{~mm}$, the cutoff position of the pinhole is located within the Airy disk of the local supplement beam. The smaller the defect size is, the more area of the Airy disk is cut off, and the more energy loss occurs. Thus, the modulation degree of the beam decreases as the defect size decreases.

As depicted in Figure 2, there exist turning points $(R=$ 1.4 and $R=2.1 \mathrm{~mm}$ ), and the intensification factors are both 1.4. At the same time, the defect sizes satisfy the expression $R=2 / 3 \times R_{\max } . R_{\max }$ denotes the defect size that has the most serious impact on the beam. As shown in Figure 3, the turning point indicates the boundary of two different intensity distributions. We will give a simple explanation for this phenomenon. With increasing defect size, the cutoff position gets closer to the first notch of the Airy diffraction pattern, generating an increasing intensity modulation in the modulation region. Moreover, according to (7), the intensity on the axis can be simplified to $I_{2}=\left|A_{2}-2 A_{2}^{\prime}\right|^{2}$, where $A_{2}$ and $A_{2}^{\prime}$ are the amplitudes of $U_{2}$ and $E_{2}^{\prime}$, respectively. With increasing $A_{2}^{\prime}, I_{2}$ drops and then increases. The intensity on the edge of the modulation region changes little. Thus, there must exist a defect $(R=2.1 \mathrm{~mm})$ that can make the intensity at the center and edge of the modulation region nearly equal (Figure 3(b)). When $R<2.1 \mathrm{~mm}$, the spatial filter plays a role in removing the modulation region. Thus, the center of the light field is displayed as a dark region. Meanwhile,

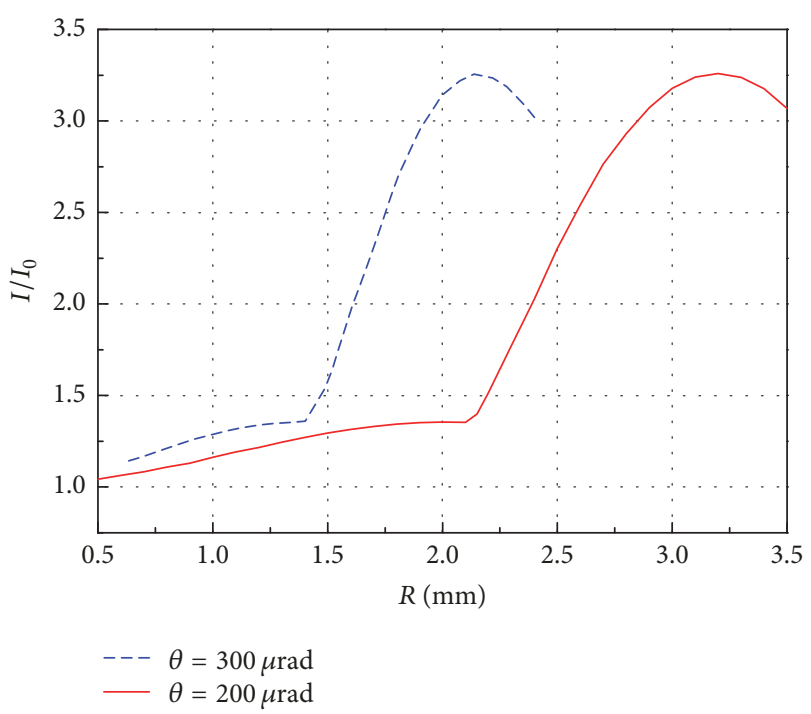

FIGURE 2: Intensification factor varying with increasing defect size at image relay plane.

the background beam is also perturbed. When $R>2.1 \mathrm{~mm}$, there is a sharp intensity modulation in the beam center that is surrounded by a circle of dark stripes. We conducted a preliminary experiment to verify the above conclusions. By changing the sizes of the defects, the distributions of the stripes can be captured by Charge-Coupled Device (CCD) (Figure 4). Compared with the simulation calculations, both results are approximately in agreement, demonstrating the credibility of the theory to a certain extent under existing experimental conditions. More accurate experiments will be carried out in the future.

The quality of the downstream beam when using the filter pinhole is shown in Figure 5. It is found that a maximal value exists along the propagation distance. The value is larger than the one at the image relay plane. Figures 6(a) and $6(\mathrm{~b})$ demonstrate the maximal value and corresponding location that evolve with increasing defect size. In Figure 6(a), we can see that the bigger the defect size, the greater the intensification factor when $R \leq 3.2 \mathrm{~mm}$. The trend is close to the linear change. Meanwhile, the corresponding location becomes gradually closer to the image relay plane, which may induce damage to optical components just placed at the image relay plane, such as the reflector in SG-II system. In addition, Figure 6(b) shows that as the size of the defect increases, restraining of characteristic high-frequency modulation by the spatial filter has been greatly weakened. Thus, the location of the maximum value gradually approaches the position of the modulation beam propagating in free space when $R>$ $3.2 \mathrm{~mm}$. Thus, $R=3.2 \mathrm{~mm}$ is a turning point in the process of changes in the maximal value location.

To ensure the quality of the propagation beam and the safety of optical components, the intensification factor is limited to 1.4 [18]. By analyzing the beam quality at the image relay plane and downstream plane, which is affected by different sizes of local defects, a rule identifying the maximum defect size for a given spatial filter that bounds the safe 
$U_{2}\left(x_{2}, y_{2}\right)$

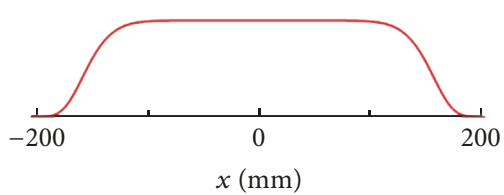

$U_{2}\left(x_{2}, y_{2}\right)$

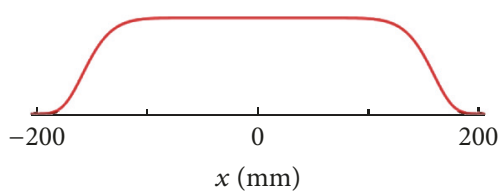

$U_{2}\left(x_{2}, y_{2}\right)$

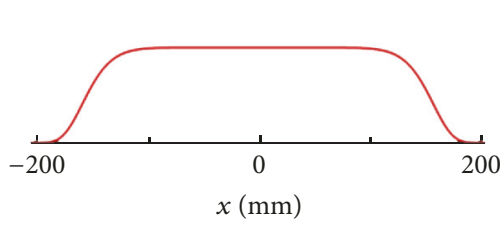

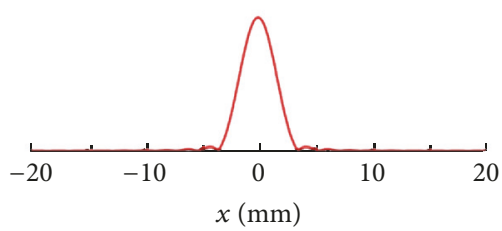

(c)

$E_{2}^{\prime}\left(x_{2}, y_{2}\right)$

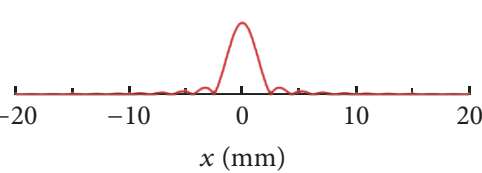

(a)

$E_{2}^{\prime}\left(x_{2}, y_{2}\right)$

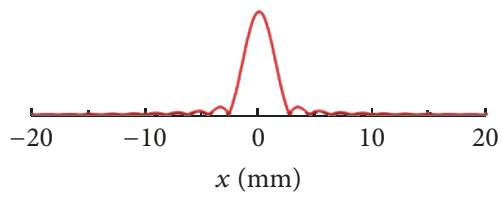

(b)

$E_{2}^{\prime}\left(x_{2}, y_{2}\right)$

FIGURE 3: Intensity profiles at image relay plane. (a) $R=1.5 \mathrm{~mm}$, (b) $R=2.1 \mathrm{~mm}$, and (c) $R=3.2 \mathrm{~mm}$.

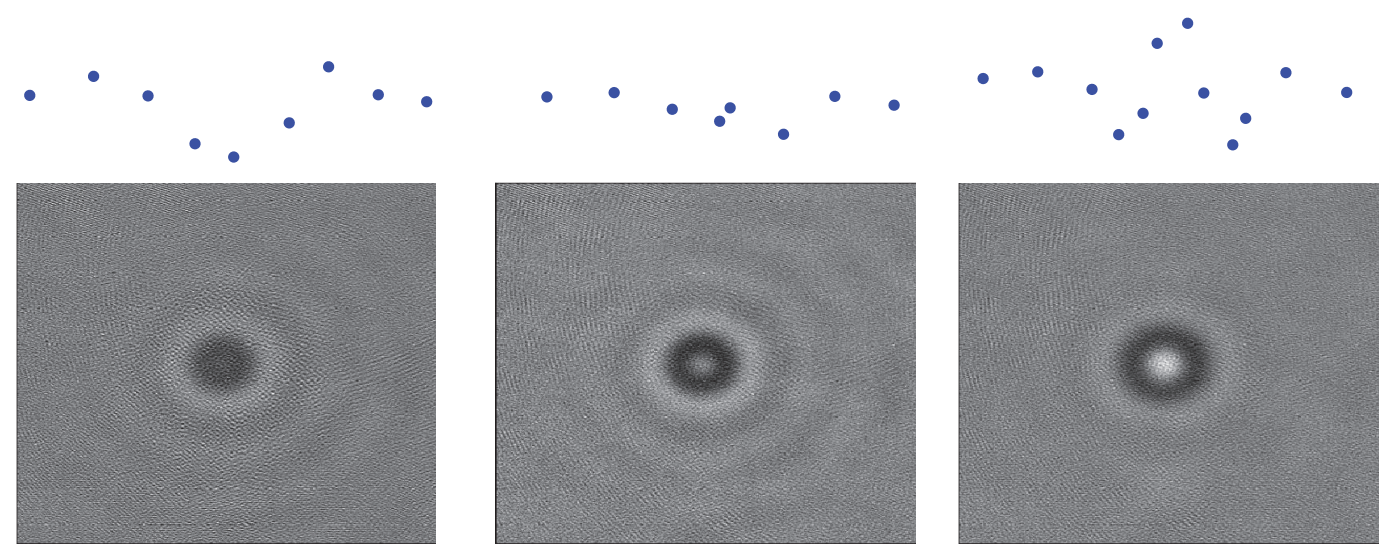

FIGURE 4: Experimental results. (a) One-dimensional intensity distributions. Blue dotted lines represent experimental results, and red solid lines represent calculation results. (b) Light intensity distributions acquired by CCD.

operating range is given by the maximum allowable defect times half-angle of spatial filter $\leq 240 \mathrm{~mm}-\mu \mathrm{rad}$. Namely, if there is a defect larger than $1.2 \mathrm{~mm}$, the optical component located at the imaging reply plane or downstream plane must be paid more attention, and some effective processes should be taken if necessary. In addition, the maximum allowable defect radiuses in different high-power laser systems are listed in Table 1, including the results in the SG-II high-power laser facility. We can observe from the table that the larger the filter pinhole is, the weaker constraints it has on the modulation 


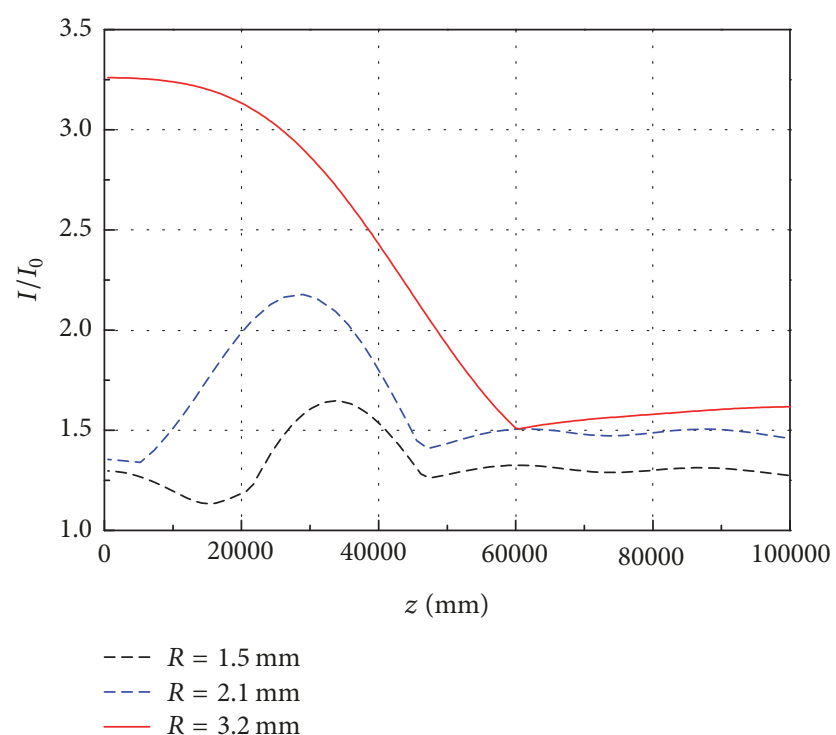

FIGURE 5: Intensification factor varying with distance from image relay plane.

TABLE 1: Maximum allowable defect radiuses in different highpower laser systems.

\begin{tabular}{lc}
\hline Filter pinhole $(\mu \mathrm{rad})$ & Defect radius $(\mathrm{mm})$ \\
\hline 80 & 3.0 \\
109 & 2.2 \\
$128[16]$ & 1.8 \\
$141[16]$ & 1.7 \\
$150[6]$ & 1.6 \\
$200[6]$ & 1.2 \\
\hline
\end{tabular}

beam, and the smaller the defect size allowed. Therefore, the constraints of defect size and the selection of filter pinhole parameters should be put forward with regard to the quality of the propagation beam.

\section{Conclusion}

The imaging property of a spatial filter system is used to minimize the intensity fluctuations. However, the spatial filter does not work effectively because of the broadening spectrum caused by local defects. The relation between the sizes of filter pinhole and local defects is presented. Based on the above, the beam quality at the image relay plane and downstream plane deteriorates significantly as the defect size increases. Ultimately, to control low-frequency beam modulation, the fatal defect size is calculated according to spatial filter parameters. We believe that these results will help in giving specifications for components and determining which defects should be eliminated during operating highpower lasers.

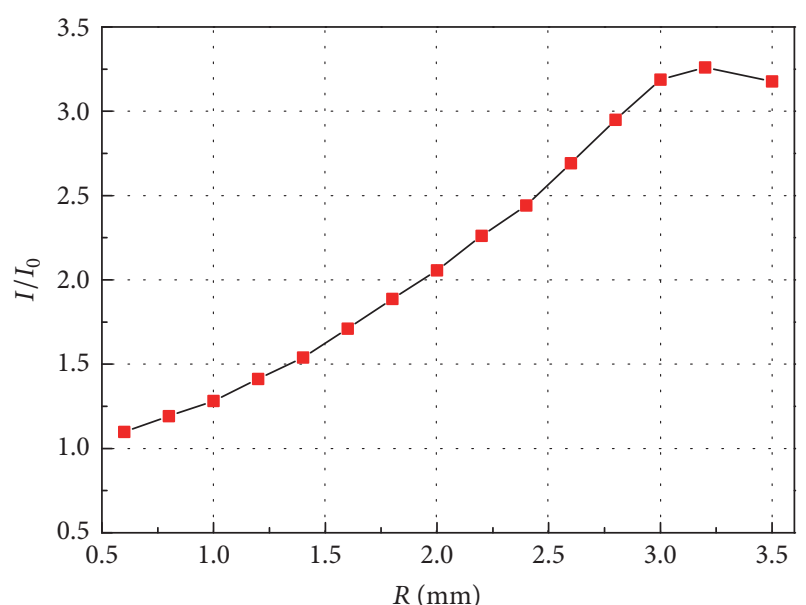

(a)

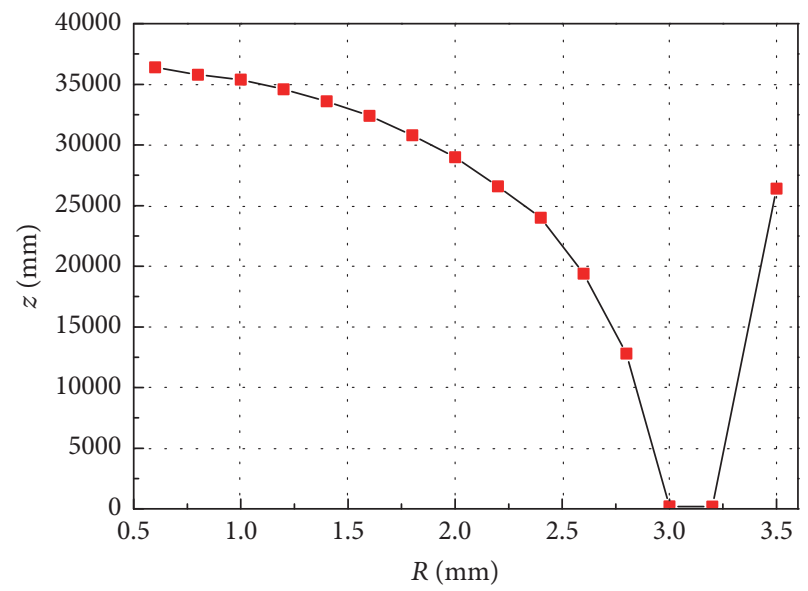

(b)

FIGURE 6: Maximum intensification factor and corresponding location varying with defect size along downstream light field: (a) maximum intensification factor and (b) corresponding location.

\section{Conflicts of Interest}

The authors declare that there are no conflicts of interest regarding the publication of this paper.

\section{References}

[1] S. Mainguy, B. Le Garrec, and M. Josse, "Downstream impact of flaws on the LIL/LMJ laser lines," in Proceedings of the LaserInduced Damage in Optical Materials, Boulder, Colo, USA, September 2005.

[2] M. L. Spaeth, K. R. Manes, and D. H. Kalantar, "Description of the NIF Laser," Fusion Science and Technology, vol. 69, pp. 25$145,2016$.

[3] B. Y. Wang, J. Y. Zhang, S. Shi, K. W. You, and J. Q. Zhu, "Effects of side subsurface defects induced by CNC machine on the gain spatial distribution in neodymium phosphate glass," High Power Laser Science Engineering, vol. 4, no. 1, 2016.

[4] H. Bercegol, G. J. Exarhos, A. H. Guenther et al., "The impact of laser damage on the lifetime of optical components in fusion lasers," in Proceedings of the XXXV Annual Symposium 
on Optical Materials for High Power Lasers: Boulder Damage Symposium, vol. 5273, Boulder, Colo, USA, 2004.

[5] D. F. Zhao, L. Wang, Z. Q. Lin, P. Shao, and J. Q. Zhu, "SG-II-Up prototype final optics assembly: optical damage and clean-gas control," High Power Laser Science and Engineering, vol. 3, 2015.

[6] M. L. Spaeth, K. R. Manes, and J. Honig, "Cleanliness for the NIF $1 \omega$ laser amplifiers," Fusion Science and Technology, vol. 69, no. 1, pp. 250-264, 2016.

[7] J. Honig, "Cleanliness improvements of National Ignition Facility amplifiers as compared to previous large-scale lasers," Optical Engineering, vol. 43, no. 12, pp. 2904-2911, 2004.

[8] P. M. Celliers, K. G. Estabrook, R. J. Wallace et al., "Spatial filter pinhole for high-energy pulsed lasers," Applied Optics, vol. 37, no. 12, pp. 2371-2378, 1998.

[9] J. E. Murray, D. Milam, C. D. Boley, K. G. Estabrook, and J. A. Caird, "Spatial filter pinhole development for the national ignition facility," Applied Optics, vol. 39, no. 9, pp. 1389-1405, 2000.

[10] J. T. Hunt, P. A. Renard, and W. W. Simmons, "Improved performance of fusion lasers using the imaging properties of multiple spatial filters," Applied Optics, vol. 16, no. 4, pp. 779782, 1977.

[11] R. J. Korniski, L. R. Gardner, K. P. Thompson, English Jr., and J. L. Miller, "Spatial filter lens design for the main laser of the National ignition facility," in Proceedings of the International Optical Design Conference, vol. 3482, Kona, Hawaii, USA, 1998.

[12] P. P. Sun, D. Liu, Y. L. Zhang, X. Y. Li, Y. Zhang, and J. Q. Zhu, "Evolution of low-frequency noise passing through a spatial filter in a high power laser system," Science China Physics, Mechanics \& Astronomy, vol. 54, no. 3, pp. 411-415, 2011.

[13] J. T. Hunt, J. A. Glaze, W. W. Simmons, and P. A. Renard, "Suppression of self-focusing through low-pass spatial filtering and relay imaging," Applied Optics, vol. 17, no. 13, pp. 2053-2057, 1978.

[14] M. Bray, A. Liard, and G. Chabassier, "Laser MegaJoule optics (I): new methods of optical specification," in Proceedings of the 1999 Optical Fabrication and Testing, vol. 3739, pp. 449-460, May 1999.

[15] Y. Gao, B. Zhu, D. Liu, and Z. Lin, "Propagation of flattopped multi-Gaussian beams through a double-lens system with apertures," Optics Express, vol. 17, no. 15, pp. 12753-12766, 2009.

[16] H. J. Liu, F. Jing, Q. Li, Z. T. Peng, and D. X. Hu, "The effect of spatial filter pinhole on output beams quality in high power laser," Journal of Yunnan University, vol. 184, no. 27, 2009.

[17] M. Born and E. Wolf, Principles of optics: electromagnetic theory of propagation, interference and diffraction of light, Cambridge University Press, Cambridge, UK, 7th edition, 1999.

[18] G. Feng and S. Zhou, "Discussion of comprehensive evaluation on laser beam quality," Zhongguo Jiguang, vol. 36, no. 7, pp. 1643-1653, 2009. 

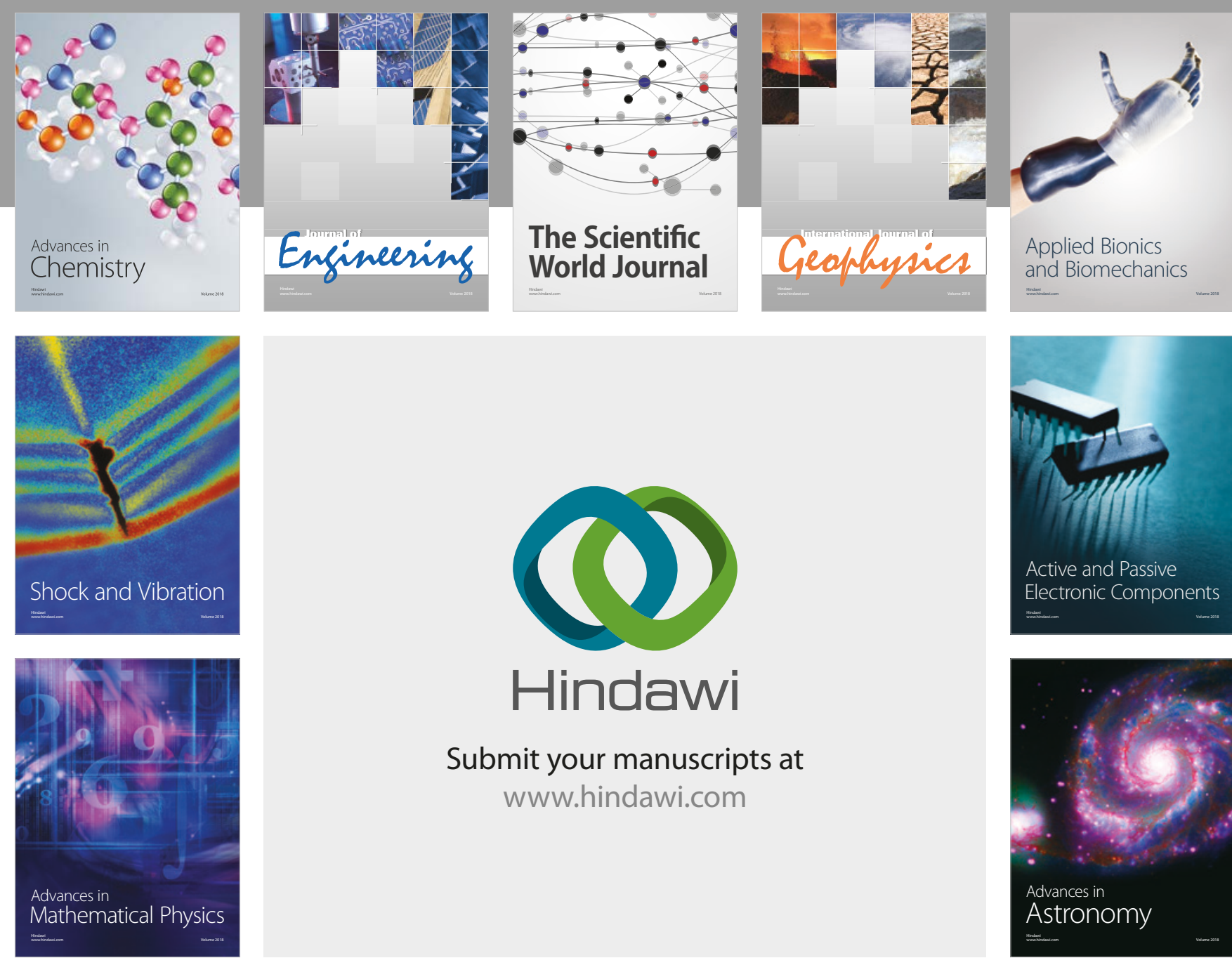

Submit your manuscripts at

www.hindawi.com

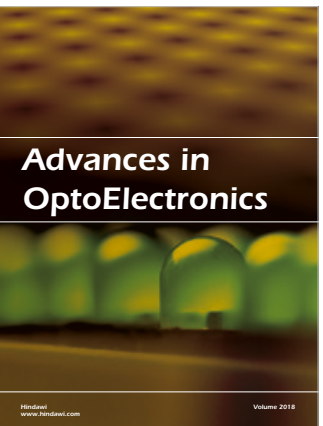

\section{Rotcting Machinery}
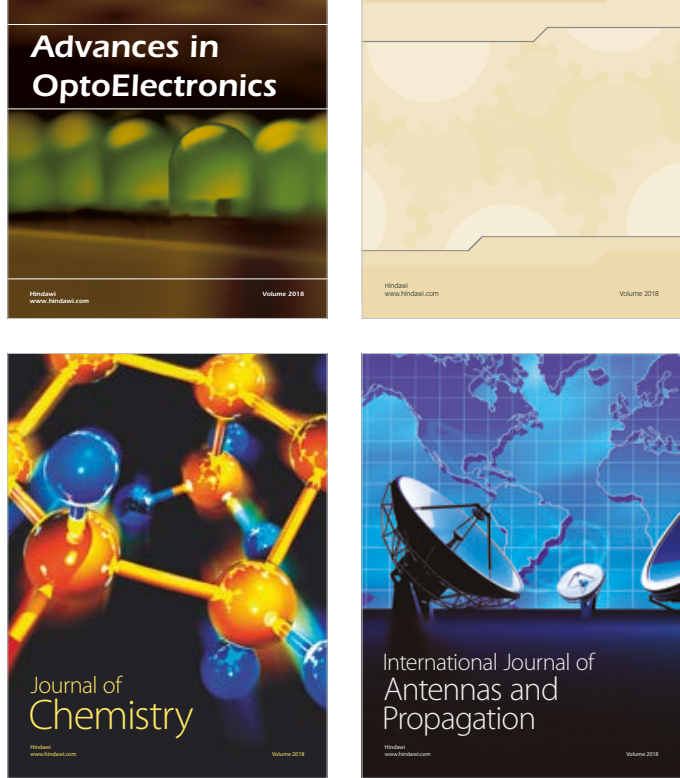

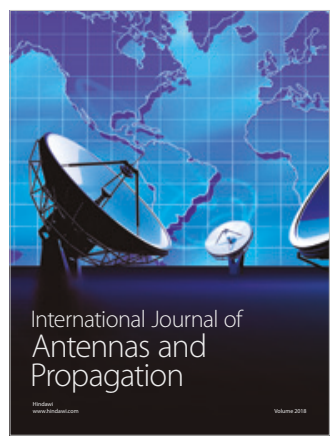

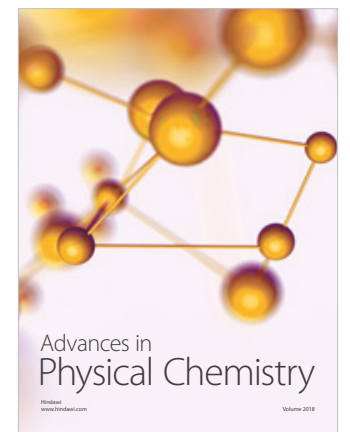

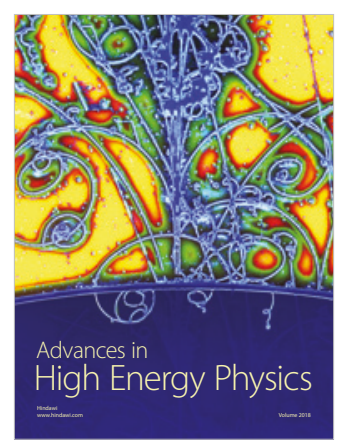

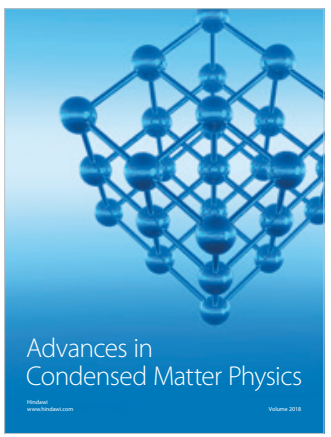

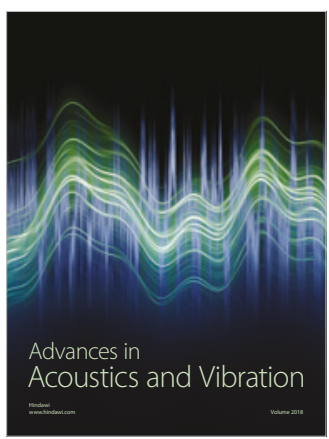

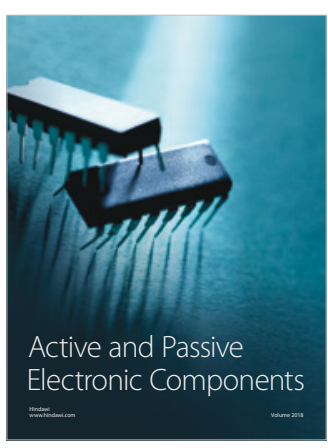
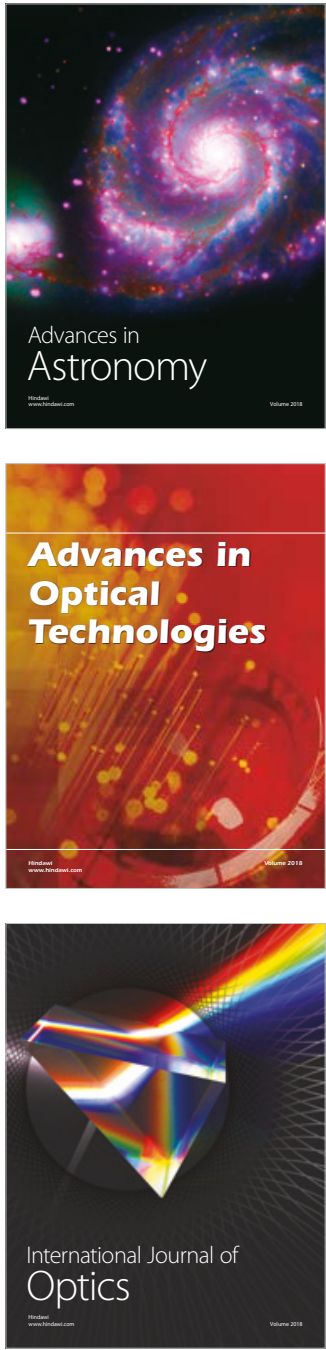